\title{
Accurate modelling for the analysis and design of liquid-crystal-based microwave devices
}

\author{
Lawrence Seddon, Richard James, Sally E. Day, \\ Member, IEEE, F. Aníbal Fernández, Member, \\ IEEE \\ Dept. of Electronic and Electrical Engineering \\ University College London \\ London, WC1E 7JE. UK \\ a.fernandez@ucl.ac.uk
}

\author{
Prafulla Deo, Member, IEEE, and Dariush \\ Mirshekar-Syahkal, Fellow, IEEE \\ School of Computer Science and Electronic Engineering \\ University of Essex \\ Colchester, Essex CO4 3SQ, UK \\ dariush@essex.ac.uk
}

\begin{abstract}
Liquid crystal substrates have been shown to provide the means to develop low-cost, reconfigurable, adaptive and tuneable microwave and MM-wave devices for mobile and wireless communication systems. However, techniques for the characterisation of materials, device fabrication and design are necessary in order to take maximum advantage of the possibilities that these materials offer. This includes appropriate modelling methods to simulate accurately the switching behaviour of the liquid crystal and the characteristics of the wave propagation through the devices, taking full consideration of the point-by-point variation of the material tensor permittivity. We describe these techniques here and show their application in the design of a meander-line phase shifter.
\end{abstract}

Keywords - Liquid crystals; microwave devices; tuneable microwave devices; reconfigurable microwave devices

\section{INTRODUCTION}

Reconfigurable, adaptive or tuneable microwave devices such as filters, couplers, antennas and phase shifters are required in modern radars as well as in mobile and wireless communication systems. Liquid crystal materials have been shown to be attractive for these applications [1-6]. Liquid crystals are liquid anisotropic materials with a tensor permittivity that can be controlled by an external, low-power, low-frequency, low-voltage signal applied across suitably placed electrodes, which can be the same metal elements used in the device for RF waveguiding. The nonuniform lowfrequency electric field thus generated causes the local reorientation of the liquid crystal material. This is reflected as a nonuniform reorientation of the permittivity tensor. In consequence, different RF field components will experience different permittivities and a proper device design should take this into account.

In order to find accurately the tensor permittivity distribution over the whole substrate, a proper modelling of the liquid crystal behaviour in the confines of the device and under the effect of a nonuniform electric field is necessary. Furthermore, material characteristics in the frequency range of operation need to be known.

In this work we show finite element software developed for

This work was funded by EPSRC (UK) under the grants EP/I003614/1 and EP/I003894/1. the material characterisation and for the analysis and design of microwave or millimetre-wave devices using a meander line phase shifter as an example.

\section{MATERIAL CHARACTERISATION AND FABRICATION ISSUES}

\section{A. Planarisation and surface treatment}

Liquid crystal device technology has been developed for displays and photonic devices operating in the optical range and its application in microwaves presents some different challenges. Optically planar surfaces are required to induce a proper liquid crystal anchoring and surface alignment but commonly used substrate materials for planar microwave devices have a large surface roughness, frequently in the range of a few microns. In consequence, the surfaces that will be in contact with liquid crystal should be appropriately coated with thick layers to reduce the surface roughness to values in the order of 0.1 or $0.2 \mu \mathrm{m}$ before alignment treatments such as rubbing are applied. Photo-alignment treatment can also be very effective in the design of complex devices if a patterned alignment is necessary to take full advantage of the liquid crystal anisotropy in relation to the local orientation of the RF electric field. For example, if the device contains lines in different directions or curves and the dominant RF field components will also change, a liquid crystal alignment that ensures the maximum effect on the dominant field components over all the device is desirable.

\section{B. Material characterisation}

Liquid crystal properties are well known in optical frequencies but few materials have been characterised in microwave frequencies. The same techniques used in optics are impractical in microwaves due to the large cell thickness required.

We take a comprehensive approach in modelling both the liquid crystal orientation and the electromagnetic field [7], [8] to develop a wideband characterisation method [9]. The permittivity tensor distribution $\quad \overline{\bar{\varepsilon}}=\varepsilon_{0}\left(\varepsilon_{\perp} \overline{\bar{I}}+\Delta \varepsilon \hat{n} \otimes \hat{n}\right)$ calculated from the liquid crystal modelling is used to calculate the microwave fields, where $\hat{n}$ is the liquid crystal director, 
$\varepsilon_{\perp}$ is the relative permittivity calculated in the direction perpendicular to the long axis of the liquid crystal molecules and $\Delta \mathcal{E}$ is the dielectric anisotropy. The desired material properties are then obtained through the comparison of the modelling and the experimental results for a simple, specially designed cell [10] within an optimisation procedure, as shown in Fig. 1.

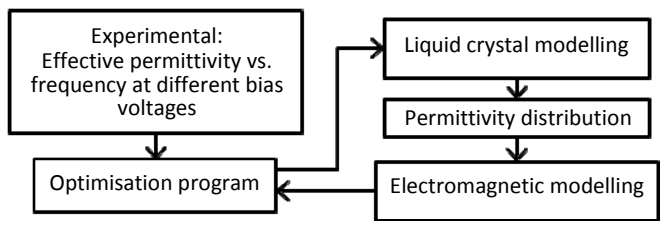

Fig. 1 Optimization procedure for liquid crystal characterisation

\section{DEVICE Modelling}

For the modelling of general devices we adopt a similar procedure used for the characterisation where the results of an accurate liquid crystal modelling are used to determine the point-by-point values of the permittivity tensor, which is then mapped onto the finite element mesh used for the electromagnetic modelling.

\section{A. Liquid Crystal Modelling}

The orientation of the liquid crystal is found by minimizing a free energy functional, which comprises elastic, electrostatic and surface contributions:

$$
\mathcal{F}=\mathcal{F}_{D}-\mathcal{F}_{E}+\mathcal{F}_{S}=\int_{\Omega}\left(f_{D}-f_{E}\right) d \Omega+\int_{\Gamma} f_{S} d S
$$

where $f_{D}, f_{E}$ and $f_{S}$ are the elastic, electric and surface energy densities. The elastic energy density is given by:

$$
f_{D}=\frac{1}{2} K_{11}(\nabla \cdot \hat{n})^{2}+\frac{1}{2} K_{22}(\hat{n} \cdot \nabla \times \hat{n})^{2}+\frac{1}{2} K_{33}(\hat{n} \times \nabla \times \hat{n})^{2}
$$

where $K_{11}, K_{22}$ and $K_{33}$ are the splay, twist and bend elastic constants of the liquid crystal material. The electric energy density is defined as $f_{E}=\frac{1}{2} \varepsilon_{0}(\nabla \phi \cdot \overline{\bar{\varepsilon}} \cdot \nabla \phi)$.

The solution of this nonlinear problem is found by seeking for the stationary values of the total free energy in (1) in a finite element implementation, alternating between the calculation of the director field and the electric potential, until convergence is reached.

\section{B. Electromagnetic Modelling}

In order to take into account the nonuniform characteristics of the liquid crystal switching, an accurate modelling of the electromagnetic wave propagation through the device is needed. The method we employ is a scattering approach based on a variational formulation of the Helmholtz equation and finds the full electromagnetic field in the device, or in specified ports, when an excitation field is applied. The device is contained in a region bounded by either metal walls or absorbing boundary conditions. The variational expression for the case of two ports is [11]:

$$
\begin{aligned}
& \mathcal{K}(\vec{E})=\frac{1}{2} \int_{\Omega}\left\{\frac{1}{\mu_{r}}(\nabla \times \vec{E}) \cdot(\nabla \times \vec{E})^{*}-k_{0}^{2} \vec{E} \cdot \overline{\bar{\varepsilon}}_{r} \cdot \vec{E}\right\} d \Omega \\
& +\sum_{k=1}^{2} \int_{S_{k}}\left\{\frac{j k_{0}}{2}(\hat{n} \times \vec{E}) \cdot(\hat{n} \times \vec{E})^{*}+\vec{E}_{t} \cdot U_{k}^{i n c}\right\} d S \\
& +\int_{\Gamma \backslash \cup S_{k}}\left\{\frac{j k_{0}}{2}(\hat{n} \times \vec{E}) \cdot(\hat{n} \times \vec{E})^{*}\right\} d S
\end{aligned}
$$

The excitation vectors $U_{1,2}^{i n c}$ are determined from the modal fields at the ports and are given by:

$$
\begin{aligned}
& U_{1}^{i n c}=-2 j k_{0} E_{t}^{M_{1}}+(R-1) \nabla_{t} E_{z}^{M_{1}} \\
& U_{2}^{i n c}=T \nabla_{t} E_{z}^{M_{2}}
\end{aligned}
$$

where the coefficients $R$ and $T$ are found by iteration. Expressions for $R$ (or $\mathrm{S}_{11}$ ) and $T$ (or $\mathrm{S}_{21}$ ) can be derived to be:

$$
\begin{aligned}
& R=\frac{\left.\int_{S_{1}} E_{t}^{M_{1}{ }^{*}} \cdot \vec{E}_{t}\right|_{z=z_{1}} d S}{\int_{S_{1}} E_{t}^{M_{1}{ }^{*}} \cdot E_{t}^{M_{1}{ }^{*}} d S}-1 \\
& T=\frac{\left.\int_{S_{2}} E_{t}^{M_{2}{ }^{*}} \cdot \vec{E}_{t}\right|_{z=z_{2}} d S}{\int_{S_{2}} E_{t}^{M_{2}{ }^{*}} \cdot E_{t}^{M_{2}{ }^{*}} d S}
\end{aligned}
$$

The modal fields $E_{t}^{M_{1}}$ and $E_{t}^{M_{1}}$ are obtained by modal analysis [9] on the surfaces $S_{1}$ and $S_{2}$ of the ports.

\section{A meander line phase shifter}

Fig. 2 shows a schematic diagram of the meander line phase shifter studied here.

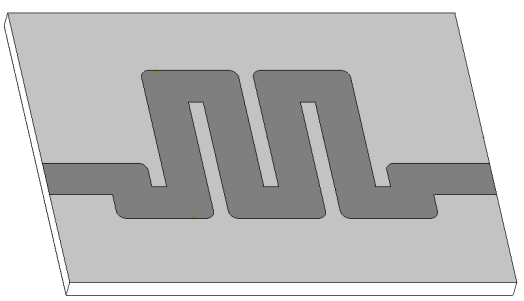

Fig. 2 Schematic diagram of the microstrip meander line phase shifter.

The approximate length of the meander line is $7 \mathrm{~mm}$ and with the material properties for GT3-23001 supplied by Merck [10] the maximum differential phase shift is approximately $40^{\circ}$ at $60 \mathrm{GHz}$, which is obtained for a bias voltage change from 0 to $14 \mathrm{~V}$. The differential phase shift as a function of voltage at 
$60 \mathrm{GHz}$ and as a function of frequency for three different values of the bias voltage is shown in Fig. 3 while Figs. 4 and 5 show the electric field and liquid crystal director distributions over the device.
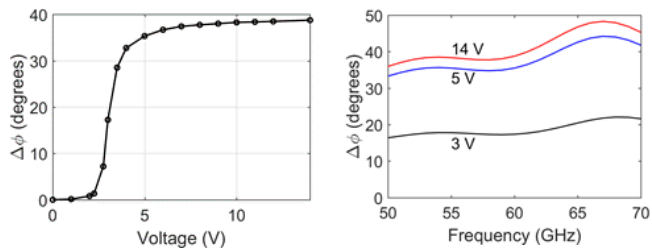

Fig. 3 Differential phase shift at $60 \mathrm{GHz}$ as a function of voltage (left) and with respect to frequency at 3 different values of the bias voltage.

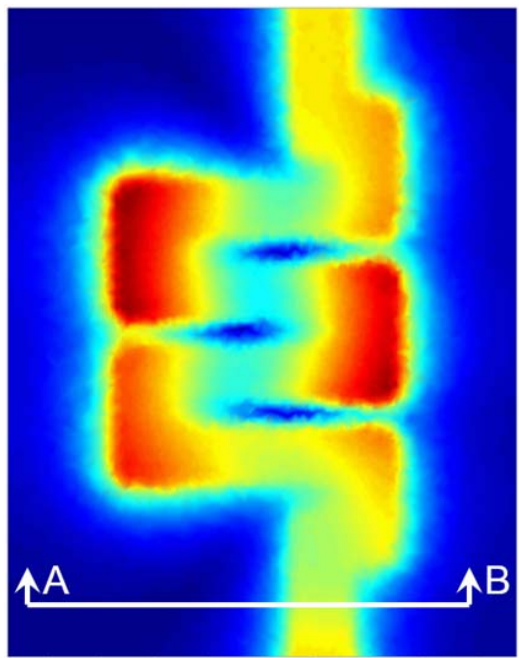

Fig. 4 Electric field (vertical component) over the middle plane in the liquid crystal substrate at $60 \mathrm{GHz}$ with a bias voltage of $14 \mathrm{~V}$.
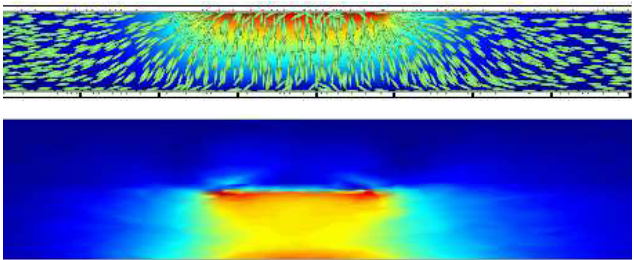

Fig.5 Director distribution (top) and electric field (bottom) over the cross section marked as AB in Fig. 4, at $60 \mathrm{GHz}$ and with a bias voltage of $14 \mathrm{~V}$.

\section{CONCLUSIONS}

A modelling procedure for the analysis and design of liquid crystal-based microwave devices has been discussed and demonstrated with an example of a meander line phase shifter. The method combines an accurate calculation of the nonuniform tensor permittivity over the whole liquid crystal substrate, via the modelling of the liquid crystal switching with the modelling of the electromagnetic wave propagation through the device when an excitation field is applied at one defined port.

\section{REFERENCES}

[1] H. Sing et al., "A $60 \mathrm{GHz}$ wireless network for enabling uncompressed video communication," IEEE Comm. Mag., vol. 64, pp. 71-78, 2008

[2] S. Bulja, D. Mirshekar-Syahkal, M. Yazdanpanahi, R. James, S.E. Day, and F.A. Fernandez, "60 GHz reflection type phase shifter based on liquid crystal," Proc. IEEEE Radio and Wireless Symp., pp. 697-699, Jan. 2010.

[3] R. Ito et al., "Fundamental properties of novel design microstrip line type of liquid crystal phase shifter in microwave region," Jpn. J. Appl. Phys., pp. 044 104-1-044 104-5, March 2012.

[4] T. Kamei, M. Yokota, R. Ozaki, H. Moritake, and N. Onodera, "Microstrip array antenna with liquid crystals loaded phase shifter," Mol Cryst Liq Cryst, pp. 167/[689]-175/[697], June 2011.

[5] K. Sahbani et al., "Coplanar liquid crystal reconfigurable phaseshifters," Mol Cryst Liq Cryst, pp. 204/[726]-212/[734], June 2011.

[6] J. Torrecilla et al. (2013). "Microwave tunable notch filter based on liquid crystal using spiral spurline technology," Microwave Opt Technol Lett, 55(10), 2420-2423.

[7] R. James, E.Willman, F. A. Fernández, and S. E. Day, "Finite-element modeling of liquid-crystal hydrodynamics with a variable degree of order," IEEE Trans. Electron Devices, vol. 53, no. 7, pp. 1575-1582, Jul. 2006.

[8] F. A. Fernández, S. E. Day, P. Trwoga, H. Deng, and R. James, "Threedimensional dynamic modelling of liquid crystal display cells using finite elements," Molecular Crystals Liquid Crystals, vol. 375, pp. 291299, 2002.

[9] R. James, F.A. Fernandez, S.E. Day, S. Bulja, M. Yazdanpanahi, and D. Mirshekar-Syahkal, "Accurate modelling for the wideband characterisation of nematic liquid crystals for microwave applications," IEEE Trans. Microw. Theory Techn., pp. 3393-3397, December 2009.

[10] P. Deo, D. Mirshekar-Syahkal, L. Seddon. S.E. Day and F.A. Fernandez, "Microstrip Device for the Broadband (15-65 GHz) Measurement of Dielectric Properties of Nematic Liquid Crystals," IEEE Trans. Microw. Theory Techn., pp. 1388-1398, April 2015.

[11] J. Jin, "The Finite Element Microwave Tunable Notch Filter Based on Liquid Crystal Using Spiral Spurline Technology Method in Electromagnetics", J. Wiley, N.Y, 1993. 